\title{
Recent Advances and Patents on Marinosomes- A Potential Nano Carrier in Pharmaceutical Technology
}

\author{
K.L. Deepthi ${ }^{*}$, S. Pravallika ${ }^{2}$ and Jagdeesh Panda ${ }^{3}$ \\ ${ }^{1 *}$ Associate Professor, Department of Pharmaceutical Technology, Raghu College of \\ Pharmacy, Visakhapatnam. \\ 2Department Of Pharmaceutical Technology, Raghu College of Pharmacy, Visakhapatnam. \\ 3Principal, Raghu College of Pharmacy, Visakhapatnam.
}

Received: 10 Oct 2020 / Accepted: 8 Nov 2020/ Published online: 01 Jan 2021 *Corresponding Author Email: deepthi.mpharmacy@gmail.com

\begin{abstract}
Currently one of the increasing interests in lipid-based delivery systems are formation of vesicular system that is passive, non-invasive and is offered for immediate commercialization. Various such systems, which have gained an utmost importance, like vesicular systems including liposomes, niosomes, pharmacosomes, transfersomes and sphingosomes. Liposomes made of phospholipids are liable to Oxidation. Marinosomes are liposomes based on a natural marine lipid extract containing high ratio polyunsaturated fatty acids and can increase stability and increases circulation lifetime [t1/2] of drug which tends to deposit in the tissues.
\end{abstract}

\section{Keywords}

liposomes, sonication, encapsulation, marine phospholipids

\section{INTRODUCTION}

The therapeutic index of former and novel drugs is increased via the rise of specificity because of targeting of medication to a selected tissue, cell or intracellular compartment, the control over pharmacokinetics, the protection of the active principle or a mixture of the above. From the last two decades, lipoidal vesicular systems are under extensive investigation as carriers for the improved delivery of a broad spectrum of drugs.[1]

Increasing interest in lipid-based delivery systems are because of following reasons like:

- Versatility of lipid excipients

- Formulation scope for selection of different drug delivery systems

- Low risk profile
- Increased oral bioavailability and reduced plasma profile variability.

- Increased permeation of those systems when used for external application.

- Formation of vesicular system that is passive, non-invasive and is offered for immediate commercialization.

- Better characterization of lipidic excipients

- High market attractiveness for products with proprietary technology

- Improved ability to handle the key problems with technology transfer and manufacture scale-up.[2]

Phospholipids are major parts of plasma membrane and organelle membranes that maintain the integrity of the cell or organelles by creating a semiimpermeable barrier from their outside 
environment. A special amphiphilic feature of phospholipids makes them suitable to be used as excipients for poorly water-soluble drugs. They get hydrated, form micelles or are organized as lipid bilayers with the hydrophobic tails lined up against one another and the hydrophilic head-group facing the water on both sides. Various such systems, which have gained an utmost importance, like vesicular systems including liposomes, niosomes, pharmacosomes, transfersomes and sphingosomes [2].

\section{Liposomes:}

Liposomes are vesicular structures with an aqueous core surrounded by a hydrophobic lipid bilayer membrane composed of a phospholipid and cholesterol. Phospholipids are GRAS (generally Recognized as safe) ingredients, therefore minimizing the potential for adverse effects. Solutes, such as drugs, in the core cannot pass through the hydrophobic bilayer however hydrophobic molecules can be absorbed into the bilayer, enabling the liposome to carry both hydrophilic and hydrophobic molecules [3]

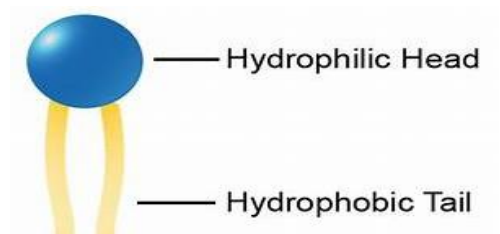

Fig.no. 1 structure of phospholipid ${ }^{[17]}$

Phospholipid chemistry:

Triacylglycerols and phospholipids contain fatty acids are the oxidizable substrates. Glycerophospholipids have two long chain fatty acid residues, esterified with a glycerol molecule that also carries a phosphate group.

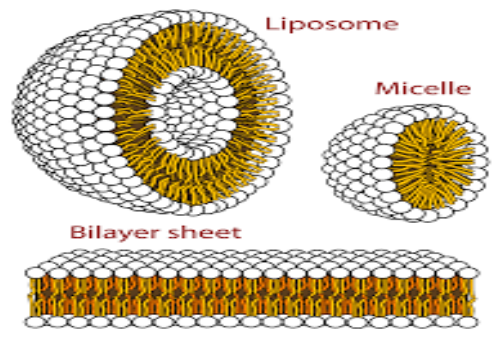

Fig.no 2 structure of liposome ${ }^{[18]}$

The phosphate is almost always linked to the SN-3 position of the glycerol molecule. The phosphate group in turn esterified with one of the organic bases, amino acids, or alcohols, which add to the hydrophilic character of the molecules. The hydrophilic group in many phospholipids includes both phosphate groups (negatively charged at the $\mathrm{pH}$ values normally found in food) and quaternary nitrogen atoms (positively charged) and is therefore classified as zwitterionic. [8]

\section{Major problems of liposomes made of} phospholipids:

1. Liposomes made of phospholipids are liable to Oxidation. Nacak et.al. studied on the oxidative stability of $\alpha$-tocopherol incorporation into liposome ratios ranged from 0.01 to $12 \mathrm{~mol} \%$.
Best oxidative stability was obtained for liposomes that contained $5 \mathrm{~mol} \% \quad \alpha$ tocopherol.[4]

2. Poor encapsulation efficiency.

\section{Marinosomes:}

Marinosomes are liposomes based on a natural marine lipid extract containing high ratio polyunsaturated fatty acids like eicosapentaenoic acid (EPA) and docosahexaenoic acid (DHA). They are not present in normal skin epidermis. However, they are metabolized by skin epidermal enzymes into antiinflammatory and anti-proliferative metabolites that are associated with a variety of benefits with respect to inflammatory skin disorders by regulating PGE2 and IL-8 production in human keratinocyte cultures. The preventing effect of Marinosomes was highly 
dependent on the lipid concentration used and the liposome mean diameter. Active and passive loading of drug, as well as complex structural rearrangements, directly depends on transmembrane $\mathrm{pH}$ gradient . All these results allowed considering Marinosomes as potential candidates for cosmeceutical and oral PUFA supplements in view of the prevention and treatment of deficiencies.[2]
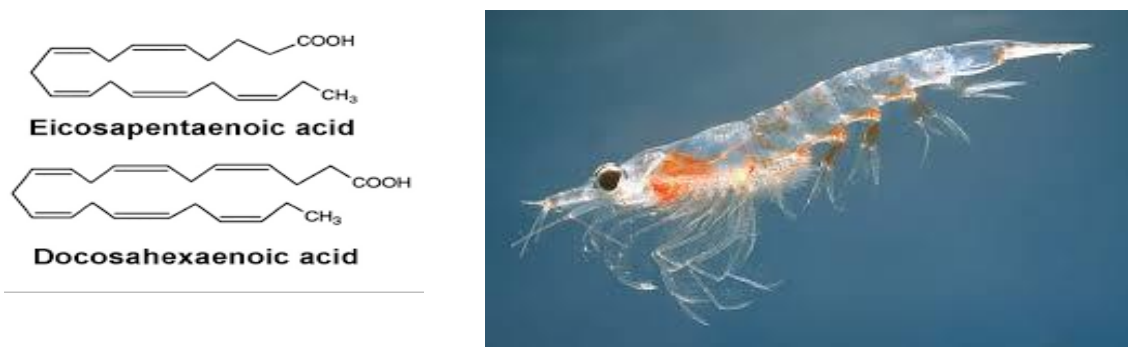

Fig.no 3: structure of marine phospholipids-EPA AND DHA ${ }^{[18]}$

Advantages of marine phospholipids:

Fig.no 5: Salmon ${ }^{16}$

\begin{tabular}{|c|c|c|c|c|c|c|c|}
\hline S.NO. & Marine species & & $\begin{array}{l}\text { Common } \\
\text { name }\end{array}$ & $\begin{array}{l}\text { Isolated } \\
\text { source }\end{array}$ & $\begin{array}{l}\text { Active } \\
\text { compound }\end{array}$ & $\begin{array}{l}\text { \% of } \\
\text { phosphatidyl } \\
\text { ethanol } \\
\text { amide }\end{array}$ & $\begin{array}{l}\text { \% Of } \\
\text { phosphatidyl } \\
\text { choline }\end{array}$ \\
\hline 1. & Aaspatopapitlata & Tuberella papillate & $\begin{array}{l}\text { Marine } \\
\text { sponge }\end{array}$ & $P E, P C$ & - & 11.6 & \\
\hline 2. & Callyspongiasp & Callyspongiid & $\begin{array}{l}\text { Marine } \\
\text { sponge }\end{array}$ & $P E, P C$ & 9.1 & 17.6 & \\
\hline 3. & Ciocalyptasp & - & $\begin{array}{l}\text { Marine } \\
\text { sponge }\end{array}$ & $P E, P C$ & 53.7 & 6.5 & \\
\hline 4. & Dysideasp & - & $\begin{array}{l}\text { Marine } \\
\text { sponge }\end{array}$ & $P E, P C$ & 35.4 & 9.0 & \\
\hline 5. & Gelliodessp & -- & $\begin{array}{l}\text { Marine } \\
\text { sponge }\end{array}$ & $P E, P C$ & 7.2 & 59.0 & \\
\hline 6. & Higginsiatethyoides & - & $\begin{array}{l}\text { Marine } \\
\text { sponge }\end{array}$ & $P E, P C$ & 5.9 & 52.6 & \\
\hline 7. & Leucettasp & Niphates digitalis & $\begin{array}{l}\text { Marine } \\
\text { sponge }\end{array}$ & $P E, P C$ & 21.6 & - & \\
\hline 8. & Mymekiodermadendyl & Leucettachagosensis & $\begin{array}{l}\text { Marine } \\
\text { sponge }\end{array}$ & $P E, P C$ & 8.2 & 54.5 & \\
\hline 9. & Niphantessp & Pink vase sponge & $\begin{array}{l}\text { Marine } \\
\text { sponge }\end{array}$ & $P E, P C$ & 7.0 & 51.8 & \\
\hline 10. & Oceanapiasp & - & $\begin{array}{l}\text { Marine } \\
\text { sponge }\end{array}$ & $P E, P C$ & 6.0 & 56.3 & \\
\hline 11. & Ptiloculisspiculifer & Tree sponges & $\begin{array}{l}\text { Marine } \\
\text { sponge }\end{array}$ & $P E, P C$ & 3.0 & 56.1 & \\
\hline 12. & Antartica krill & Euphansiasuperba & Fish & \multicolumn{4}{|c|}{ polyunsaturated fatty acid $12.5-21$} \\
\hline 13. & Salmon & $\begin{array}{l}\text { Salmo } \\
\text { salar Linnaeus }\end{array}$ & fish & PC & --- & $38-75$ & \\
\hline
\end{tabular}

Table.1. sources of various marine phospholipids ${ }^{[12,14]}$ 
- Marinosomes are stable at various $\mathrm{pH}$ and at elevated temperatures range compared to liposomes.

- Marinosomes can entrap food ingredients (enzymes, flavors, minerals, and vitamins) for human and animal nutrition example in nutraceutical.

- Marine phospholipids are considered as an attractive material for development of liposome as an orally polyunsaturated fatty acid supplement for its high lipid bioavailability.

- Marinosomes increases circulation lifetime [t1/2] of drug which tends to deposit in the tissues.

- Marinosomes protects the metabolic degradation of drug.

- Marinosomes altered tissue distribution of drugs with enhanced uptake in organs rich mononuclear phagocytic cells in liver, spleen, and bone marrow and decreases uptake in kidney, myocardium and brain [11]

Patented methods for the extraction of lipids from marine sources: ${ }^{[15]}$

- U.S. Pat. No. 4,331,695 describes a method using pressurized Solvents which are gaseous at room temperature, Such as propane, butane or hexane. The extraction is per formed at preferred temperatures of 15 to $80^{\circ}$ $C$ on finely divided animal products. The extracted oils are then made to precipitate under high pressure and elevated temperatures of 50 to $200^{\circ} \mathrm{C}$. However, hexane is a poor extraction Solvent for marine animals. Such as krill. Furthermore, the high temperatures used in the precipitation Step negatively alters the lipids.

- Canadian Patent Application 2,115,571 describes a method for extracting oils from various brown and read algae species. The method provides for example Soxhlet extraction using nearly pure ethanol for 40 hours.

- U.S. Pat. No. 5,006,281 describes a method for extracting oil from marine and aquatic animals Such as fish. The marine and aquatic animal is first treated with an antioxidant compound, finely divided and centrifuged to Separate the oil phase from the aqueous phase and Solid phase. The oil phase is then further treated with antioxidant to remove undesirable odour or taste.

- Canadian Patent 1,098,900 describes a method for extracting oils from krill. The method involves emulsifying fresh or defrosted krill in an aqueous medium. The oil fraction is recovered by centrifugation.

- $\quad$ Folch in the article published in the year 1957 in J. biol. Chem. 226: 497-509 "A simple method for the isolation and purification of total lipids from animal tissues' proposes an extraction method using chloroform and methanol. This method is not commercially feasible because of the toxicity of the Solvents involved.

\section{FACTORS EFFECTING MARINOSOMES:}

pH:

This physicochemical instability of marinosomes was even more pronounced in acid storage conditions, i.e., $\alpha$-tocopherol incorporation into liposome membranes accelerated the structural rearrangements and increased the rate of phospholipid hydrolysis. In particular, giant vesicles incubated at $\mathrm{pH} 1.5$ underwent complex irreversible shape transformations including invaginations. In parallel, the absorption rate of $\alpha$-tocopherol was measured in lymph-cannulated rats when atocopherol was administrated, as liposome suspension or added to sardine oil, through a gastrostomy tube. $\alpha$-Tocopherol recovery in lymph was increased by almost threefold, following liposome administration [4]

Effect of surfactants:

The decrease in the micro-viscosity of the liposome membrane, that is a fluidification, as well as of an increase in $\tau$, which provokes a similar effect. Nevertheless, the quenching studies show that the fluorescence intensity of the probe decreases in the presence of surfactant, which, considering the quenching phenomenon to be of a collision nature, means that the lifetime of the probe decreases observed an increase in micro viscosity of phospholipid vesicles at low concentrations of surfactants, which suggests an improvement in the packing of phospholipids molecules by the insertion of surfactants into the outer bilayer. It is known that incorporation of the surfactant is accompanied by formation of a free volume close to the amphiphilic, which can be eliminated by trans-gauche isomerization, bending and /or inter digitations of the lipid chains Ptation phenomena can result on a higher packing density of the lipid molecules and can explain, at least in part, the These vesicle size decrease observed for the first Triton X-100 concentrations, as well as the anomalous dequenching and membrane rigidifying described.

\section{Surface charge:}

Liposomes containing charged surfactants exhibited a decrease in the hydrodynamic shear diameter, 
attributable mainly to a reduction in the electrostatic charge of the headgroups that causes a bilayer condensation. For higher amphiphile concentrations, a diameter increase starts to occur, probably due to an excess of charge, which causes repulsion of the head [10].

Diameter:

Marinosomes suspension acidification led to an instantaneous vesicle aggregation and to complex morphological bilayer rearrangements (8). Different mechanisms have been advocatto explain the liposome size decrease, including the osmotic shock occurring at acid $\mathrm{pH}$ and/or membrane vesiculation attributable to chemical lipid modifications. Increasing the temperature to $37^{\circ} \mathrm{C}$ seemed to speed these processes as shown by the decrease in turbidity and the formation of small lipid particles Simultaneously, chemical modifications, in particular oxidation and hydrolysis, occurred which may also proceed to structural changes.[7]

\section{Effect of bile:}

Micelle formation with the bile salts effect the marinosome preparation. The solubilization process was visualized by the turbidity variations measured as a function of bile salt addition. Since detergent was externally added to preformed liposomes, it was essential to work with bile salts completely equilibrated within the lipid structures. Twenty-four hours were required to obtain constant, quasi-null turbidity values for the mixed micelle systems (result not shown), indicating that equilibrium was slowly reached compared to large unilamellar vesicles [6].

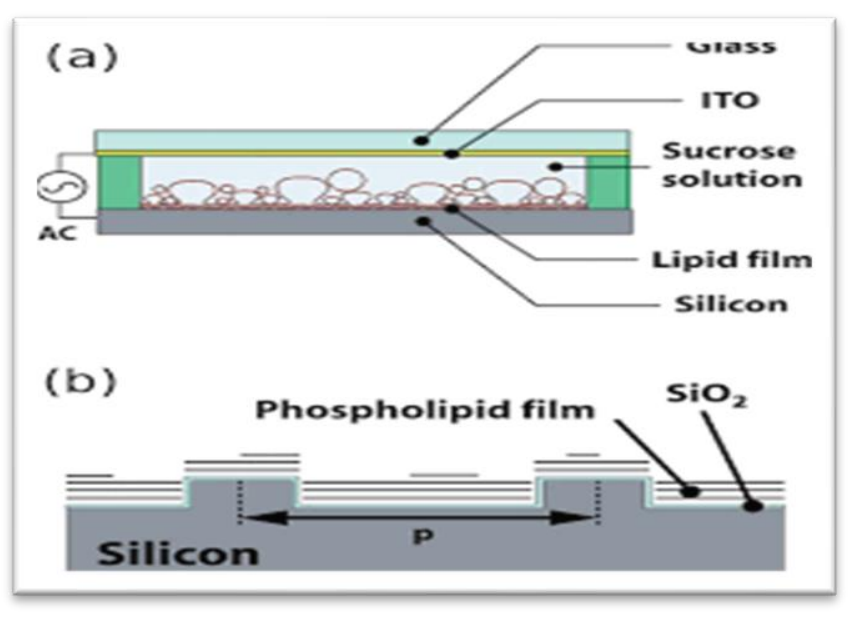

Figure 5 Electro formation ${ }^{[20]}$

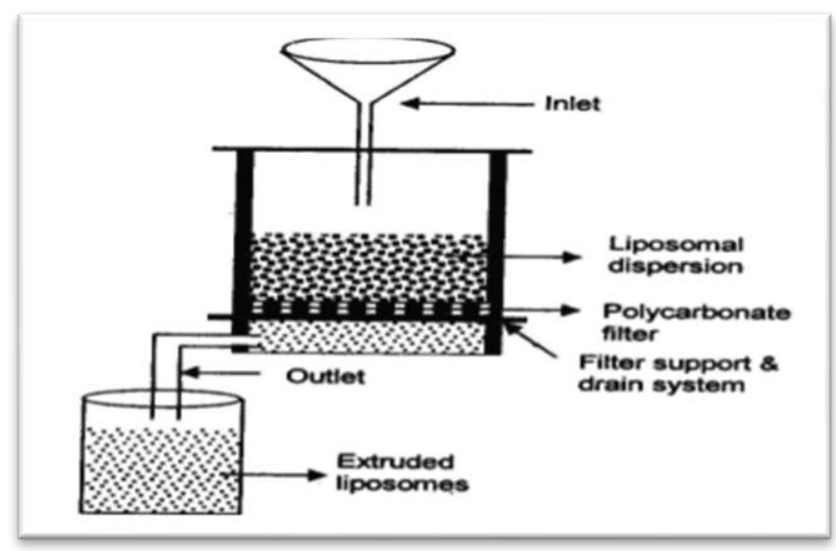

Figure 6 Extrusion method ${ }^{[21]}$

\section{Methods of preparation:}

\subsection{Electro formation}

Giant vesicles were prepared using the electro formation procedure. Six nanograms of marine lipids dissolved in chloroform/ methanol (9:1 v/v) were deposited in six locations on two platinum wires introduced into the experimental chamber as shown in figure. The organic solvent was removed by evaporation under vacuum for $1 \mathrm{~h}$. The chamber was slowly filled with Tris buffer $(\mathrm{pH} 7.4)$ to prevent lipid dispersion. A $10-\mathrm{Hz}$ electric field was applied increasing from a low (50 mV mm-1) to high (800 mV 
$\mathrm{mm}-1$ ) amplitude in $40 \mathrm{~min}$. Then, the chamber was left overnight at $10 \mathrm{~Hz}, 800 \mathrm{mV} \mathrm{mm}$. Vesicle dispersion was promoted by the application of a low frequency field $(4 \mathrm{~Hz})$ followed by a gentle hand shaking of the cell. [4]

Extrusion:

Filtered marinosomes were obtained froma lipid suspension (3mg ml ${ }^{-1}$ ) in Hepes buffer \{2-[4-(2 hydroxyethyl)piperazin-1 yl]ethanesulfonic acid $\}(\mathrm{pH}$ 7.4) after a single extrusion through polycarbonate filters of 5-mm pore size (Millipore) $[9,10]$. Lipid loss due to filtration was checked using phosphorus and enzymatic assays.[4]

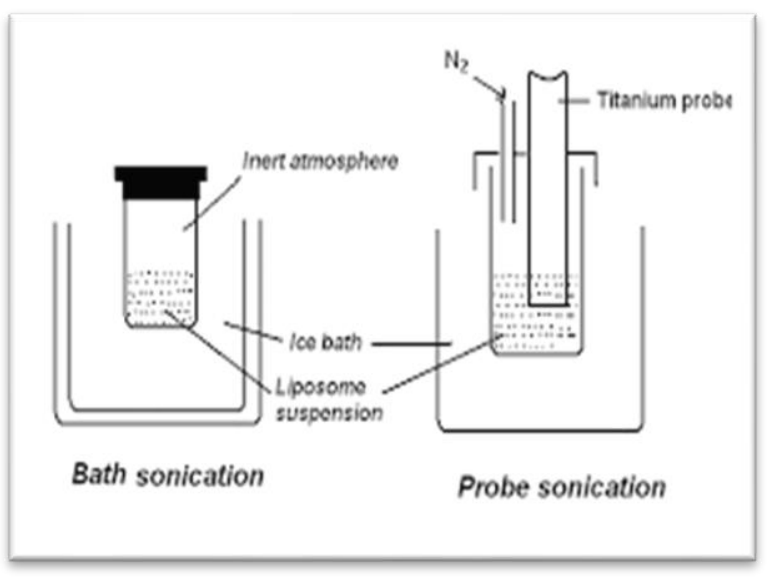

Figure 7: Sonication [23]

\section{Sonication:}

Marinosome preparation started with solvent removal from the marine lipids by gently passing nitrogen (99.99\%) over the solution. After removal of the solvent, the phospholipids were kept under vacuum for 1 hour. The dried film of phospholipids was dissolved in a $5 \mathrm{mM}$ pH 5.5 MES (2morpholinoethanesulfonic acid, Sigma) buffer to a concentration of $30 \mathrm{mg} / \mathrm{ml}$. MES buffer is known as a "Good type" buffer, and it was chosen for several reasons. It does not bind iron as for example phosphate buffers and has a very low solubility in nonpolar solvents. As most of our oxidation experiments were performed at $\mathrm{pH} \approx 5.5$ (observed highest oxidation rate), MES buffer was also a good candidate as the effective $\mathrm{pH}$ is given as 5.5-6.4 ( $p K=6.1$ ). To get phospholipids dispersed in aqueous media as bilayer structured liposomes several methods such as hand shaking, sonication, membrane extrusion etc. can be used (New, 1990). The probe sonication method was chosen because only a short time was needed to disperse phospholipids in the buffer. The solution was sonicated for 15s x 10 times with an MSE Ultrasonic Disintegrator Mk2 (MSE Scientific instruments, Sussex, England). Each sonication treatment was followed by a 30s break and cooling in ice was used to avoid temperature increase. A total sonication time of $2.5 \mathrm{~min}$ was enough to disperse all phospholipids in buffer. When sonication time of 5 min was used to make liposomes, no decrease in particle size distribution was observed. According to the literature increased sonication time decrease oxidative stability of liposomes (Klein, 1970, Genot et al., 1999). Due to this a sonication time of $2.5 \mathrm{~min}$ was chosen as the optimal time. [8]

Thin drug-lipid film hydration method

Curcumins were prepared by reflux followed by thin drug-lipid film hydration method, which mentioned in detail in our previous study with some modification (Ibrahim et al., 2017). Krill lipids were taken with different weights $(10,15,20$, and $30 \mathrm{mg})$ in a glass bottle and dissolved in chloroform/methanol mixture $(2 / 1, v / v)$. Curcumin (1mg) was added to the lipid solution and the mixture was refluxed (tightly close the glass bottle and stir at a temperature of $50^{\circ} \mathrm{C}$ for $2 \mathrm{~h}$ ). The resultant clear solution was evaporated to provide thin lipid films that were placed under high vacuum for at least overnight to remove the residual solvent. The dried drug-lipid film was hydrated in HEPES buffered Saline (HBS) (20mM HEPES, $150 \mathrm{mM} \mathrm{NaCl}$, $\mathrm{pH} 7.5$ ) to form multilamellar vesicles (MLVs). For the CURMs prepared using extrusion method, the MLVs were extruded (Mini-extruder, Avanti Polar Lipids, Alabaster, AL, USA) 10 times through two Whatman Nucleopore TrackEtched polycarbonate membranes (200nm×10; GE Healthcare Life Sciences; Chicago, IL, USA) at $50^{\circ} \mathrm{C}$ to decrease their sizes. For the CURMs prepared using sonication, the MLVs were sized using an ultrasonic homogenizer (UH-50; SMT Company, Tokyo, Japan). The prepared formulations were cooled on ice for $15 \mathrm{~min}$ and unencapsulated curcumin was removed by centrifugation (1500g, 
$\left.4^{\circ} \mathrm{C}, 15 \mathrm{~min}\right)$. The mean size and polydispersity index (PDI) were determined using a dynamic light scattering instrument (ZetaSizer Nano-ZS; Malvern Instrument Ltd., Malvern, U.K.). Zeta potentials were measured using ZetaSizer Nano ZS90.[11]

\section{EVALUATION OF MARINOSOMES \\ Physical evaluation}

PH The $\mathrm{pH}$ of marinosomes suspension was carried out using ph meter with a combined glass calomel electrode at $20^{\circ} \mathrm{C}[4,6]$

Optical density Turbidity measurements can be performed at $37^{\circ} \mathrm{C}$ in a thermo stated cell support using a Spectrophotometer to follow marinosome stability as a function of time. $[4,6]$

Surface charge the zeta meter was used to measure the zeta potential of marinosomes. it is done at phospholipid concentration $6 \mathrm{mg} / \mathrm{ml}$ the zeta potential of $(\mathrm{mV})$ of marinosomes was determined from electrophoretic mobility measurements [8]

Particle size:

Size distribution, i.e., mean diameter and polydispersity index, was determined by quasielastic light scattering (QELS) and granulometry measurements. The apparatus used for QELS measurements consisted of a goniometer (BI-200SM; Brookhaven Instrument, Holtsville, NY), a photomultiplier tube (EMI PM-28B), a digital correlator (BI-9000AT; Brookhaven Instrument) and a krypton ion laser (Innova 90; Coherent, Santa Clara, CA). The scattering cell was immersed in a refractive index matching fluid whose temperature was controlled at $25 \pm 0.1^{\circ} \mathrm{C}$. For each sample, data were acquired, typically for aduration of $10 \mathrm{~min}$, for three runs at angles ranging from 30 to $150^{\circ} \mathrm{C}$. Size measurements were also performed using a photon correlator spectrophotometer.[6]

Encapsulated efficiency and trapped volume:

These are determined by the rate and amount of entrapped volume of water-soluble agents in aqueous compartment.

Drug release:

The drug release from the marinosome is given by the invitro diffusion cell [8]

\section{CHEMICAL CHARACTERIZATION}

Marinosomes micellization by bile salts_Vesicle bilayer dissolution by bile salts was followed measuring the turbidity at $400 \mathrm{~nm}$. Lipid-bile salt mixtures were prepared by adding defined volumes of a concentrated bile salt solution $(650 \mathrm{mM}$ calculated with an average molecular weight of 481 $\mathrm{g} \mathrm{mol}-1)$ to a liposome suspension with [lip]tot ranging from 0.3 to $0.8 \mathrm{mM}$. After stirring, the mixture was allowed to equilibrate until stable turbidity measurements were obtained. Solubilization was performed at $37^{\circ} \mathrm{C}$, (i) on liposomes prepared in HEPES buffer and (ii) on liposomes that were first incubated $3 \mathrm{~h}$ in acid medium $(\mathrm{pH} 1.5)$ and then neutralized to get a final $\mathrm{pH}$ suspension at 7.4. For each [lip]tot, a solubilization curve was obtained by plotting OD as a function of total bile salt concentration ([BS]tot). The solubilization point, corresponding to the bile salt amount required to completely transform the lipid bilayers into mixed micelles, was determined by the point at which additional detergent only slightly affected the suspension turbidity value, i.e., where OD was quasi-null. At this solubilization point, the concentration of bile salt molecules that are not associated with the lipids ([BS]bulk) and the bile salt to lipid molecular ratio in the mixed micelles ([BS/lip] mic) are given by the following equation.

[BS]tot $=$ [BS]bulk + [BS/lip] mic * [lip]tot [1]

Using a linear regression analysis, [BS]bulk was found as the extrapolation to zero of the total lipid concentrations, and [BS/lip] micfrom the slope of the lines.[6]

\section{Marinosome Lipidhydrolysis:}

Lipid hydrolysis in different lipid aggregates, i.e., bilayers and mixed lipid-bile salt micelles, was followed by titration with $0.01 \mathrm{~N} \mathrm{NaOH}$ solution of the fatty acids liberated at $37^{\circ} \mathrm{C}$ using a pH-stat (Radiometer). Total lipid concentrations varied from 15.0 to $150.0 \mathrm{mM}$, which in terms of PL represented concentrations([PL]tot) ranging from 10.0 to 103.0 $\mathrm{mM}$. Initial liposome preparations were diluted in buffer (10 mM HEPES, $145 \mathrm{mM} \mathrm{NaCl} 5 \mathrm{mM} \mathrm{CaCl}$, pH 8) prior to enzyme addition. Then, liposomes were solubilized by addition of the required amount of bile salts in order to obtain mixed micelles. The hydrolysis reaction was initiated under stirring by PLA2 addition (10 IU) to $2.5 \mathrm{~mL}$ of liposome or mixed micelle preparations. The initial velocity $(\mathrm{Vi})$, defined as the micromoles of fatty acids liberated per minute, was determined from the initial slope of the titration curves of the hydrolysis experiment.[6]

\section{Chemical analysis of marinosome membranes:}

Liposome dispersion (2 $\mathrm{ml} ; 1.5 \mathrm{mg} \mathrm{ml}^{-1}$ ) was incubated in Hepes buffer at acid (1.5) and neutral (7.4) $\mathrm{pH}$, during 0, 3 and $24 \mathrm{~h}$. Lipid suspensions were directly submitted to Folch extraction and the lipids recovered in chloroform/methanol $(2: 1 \mathrm{v} / \mathrm{v})$. A twodimensional thin layer chromatography (TLC) was performed using the following solvents: in the first direction chloroform/acetone/methanol/acetic acid/water (50:20:10: 10:5 v/v/v/v/v) and in the second direction butanol/acetic acid/water (60:20:20 v/v/v). The different diacyl phospholipid and lysophospho lipid classes were visualized by 
exposing the plates to iodine vapor. The different spots were scrapped and analyzed for total phosphorus determination. Cholesterol and triglyceride contents were determined by enzymatic assays using standard kits (Boehringer Mannheim, France). [4]

\section{Marinosome oxidation}

Aldehydes as secondary lipid oxidation products were analyzed by static head space gas chromatography (GC) [15]. In a sealed vial, liposomes (1 mg) were incubated for 1 day in $1 \mathrm{ml}$ Hepes buffer at different $\mathrm{pH}$.The $\mathrm{GC}$ system consisted of a gas chromatograph (Carlo Erba GC 8000)provided with an automatic split injector (Carlo Erba HS 800) and a flame-ionization detector maintained at $250^{\circ} \mathrm{C}$. For GC separation, a $30 \mathrm{~m} \times 0.32 \mathrm{~mm}$ internal diameter column coated with $1 \mathrm{~mm}$ thickness DB1701 was used applying hydrogen as carrier gas. The temperature was maintained at $40^{\circ} \mathrm{C}$ for $5 \mathrm{~min}$ then increased to $200^{\circ} \mathrm{C}\left(10^{\circ} \mathrm{C} \mathrm{min}{ }^{-1}\right)$. Data integration was performed using a Chromjet SP400 integrator (Spectra-Physics). Quantification of the different aldehydes formed upon lipid peroxidation was carried out with calibration curves using pure aldehyde standard solutions (hexanal, pentanal, propanal).[6]

\section{$\alpha$-Tocopherol analysis:}

$\alpha$-Tocopherol present in the liposome membrane and in the lymph was quantified by highperformance liquid chromatography (HPLC) on the corresponding lipid extracts. The HPLC system consisted of an HPLC pump (Spectra system P4000) and an automatic sample injector (Spectra system AS3000). A column (Ultrasphere ODS, $25 \times 0.46 \mathrm{~cm}$, Beckman) was used with methanol as mobile phase

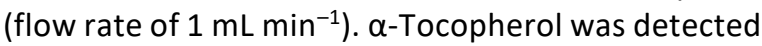
by fluorescence spectrophotometry (excitation wavelength $292 \mathrm{~nm}$ : emission wavelength $330 \mathrm{~nm}$ ). Quantification of atocopherol was carried out using a calibration curve made with pure $\alpha$-tocopherol standard solutions.[5].

\section{BIOLOGICAL CHARACTERIZATION}

Animals and surgical procedures: Official French regulations for the care and use of laboratory animals were followed. Male Wistar rats weighing 250-300 g was obtained from Elevage Janvier (SaintBerthein, France). They were housed for $1 \mathrm{wk}$ in a controlled environment, with constant temperature and humidity. They were fed a fat-free diet (UAR, Epinay, France) and allowed free access to water until $24 \mathrm{~h}$ before the surgery. Under pentobarbital anesthesia ( $0.05 \mathrm{mg} \mathrm{g}^{-1}$ body $\mathrm{wt}$ ), a polyethylene catheter (i.d. $0.86 \mathrm{~mm}$, o.d. $1.27 \mathrm{~mm}$; Biotrol, Paris, France) was inserted into the main thoracic lymph duct of each rat for collecting the lymph $(37,38)$. After surgery, the rats were placed in individual restraining cages in a warm environment with tap water freely available. A few hours after the surgical procedure, $0.4 \mathrm{~g}$ of lipids (liposome suspension or sardine oil) was administered through a gastric feeding tube followed by $1 \mathrm{~mL}$ water in the case of sardine oil. The lymph was collected for $24 \mathrm{~h}$ without fractionation in tared tubes maintained in an ice bath. During the collection period, lymph flow averaged $0.6 \mathrm{~mL} \mathrm{h-1.} \mathrm{Total} \mathrm{lipids} \mathrm{of} \mathrm{lymph} \mathrm{were}$ immediately extracted using the method of Folch et al. (35), and $\alpha$-tocopherol content was quantified. At least five cannulated rats were used for each lipid ingestion condition studied.

\section{APPLICATIONS OF MARINOSOMES:}

\section{In cancer chemotherapy:}

Curcumin is encapsulated in krill lipids-based liposomes (marinosomes) to develop a potential anticancer therapy from low-cost and readily available nutraceuticals. Reflux followed by thin drug-lipid film method is used successfully to incorporate the drug into the liposomal membrane at high encapsulation efficiency. The curcuminloaded marinosomes (CURMs) showed a powerful antioxidant activity $(\mathrm{EC} 50 \fallingdotseq 4 \mu \mathrm{g} / \mathrm{mL})$. Additionally, CURMs exhibited good physicochemical and oxidative stability after eight weeks' storage at $4^{\circ} \mathrm{C}$.

\section{Marinosome in drug delivery}

Marinosomes mediated drug or gene delivery to selected tissues or cells is a high promising approach for improving controlled or targeted delivery. an interaction of marinosomes with tissues at the cellular level is a highly complex phenomenon the main interactions processes believed to operate independently or in a combination are endocytosis fusion, adsorption and lipid transfer. similarly, the intercellular microtubules and other vesicular motor proteins and their decisive role in numerous trafficking events including endocytosis and transcytosis, have revolutionized the approaches of marinosome interaction with the target cells at the cellular and intracellular levels [12]

Several modes of drug delivery applications can be proposed for the marinosomal systems for some of the opportunistic pathogen caused diseases. the major ones are enhanced drug solubility.

Eg: Amphotericin B,minoxidil, paclitaxel, cyclosporin Marinosome in Gene delivery:

Protection of sensitive drug molecules cytosine arabinose, DNA, RNA, antisense oligonucleotides, riboenzymes was possible with marinosome preparation. 
Marinosomes in Ophthalmic Delivery:

A major problem in ocular therapeutics is the delivery of an optimal drug concentration at the site of action. The ocular drug bioavailability is often modified by the physical and chemical properties of a drug as well as by physical properties of the vehicle in which the drug is placed. Thus, selection of vehicle has been limited to the liquid and semisolid varieties, principally because of the anatomical contract of the conjunctival sac and the sensitivity of cornea to foreign objects. Amongst various vehicles and carriers, marinosome have gained considerable attention for ocular drug delivery. marinosomes offer advantage over most ophthalmic preparation in being completely biodegradable and relatively nontoxic in parallel, the first toxicology file indicated a good skill and eye tolerance towards marinosomes. A potential advantage of marinosomes is their ability to intimately contact with the cornea and conjunctival surface, thereby increasing the probability of ocular drug absorption. The ability is especially desirable for drugs that are poorly absorbed, for e.g., drugs with low partition coefficient or those with medium to high molecular weight. Marinosomes may be used as vehlicles for sub-conjunctival and intra virtual injection of both cytotoxic drugs and antibiotics in the treatment of ocular proliferative disorders [12]

Marinosomes in the delivery of enzymes:

Marinosomes may be used in the delivery of enzymes like streptokinase, protease. Marinosomes encapsulated enzymes may be used in the treatment of several enzymes deficiency diseases like albinism caused by deficiency of tyrosinase, alkaptonuria caused by deficiency of homogentisic acid oxidise.[12]

\section{Marinosomes For Delivery of Vitamins}

Marinosomes may be used as delivery system of both lipids and hydro soluble compounds, appear to be potential candidate as artificial diets for e.g., fat soluble vitamin like vitamin E. [12]

Marinsomes are carriers for vaccines:

Studies on cardiolipin (naturally occurring lipids) revealed the importance of bilayered structures in the production of antisera and concluded that liposome in fact responsible for the antigenicity of these lipids (like naturally occurring lipids) thus marinosomes also have an adjuvant effect up on protein antigens [12]

Marinosomes use in cosmetics.

A great interest of using the marinosomes in skin gels or skin creams has been increased and generated in the field of cosmetics. Vegetable phospholipids are widely used for topical applications in cosmetics and dermatology because of their high content of esterified essential fatty acids, especially linoleic acid which increases the barrier function of the skin and decreases water loss within a short period of time after its application. Soya phospholipids and vegetable phospholipids are used as they can form liposomes and their surface activity. These are ideal source for the transport of linoleic acid into the skin. $[8,12]$

Lautenschlager et al. discussed the potential use of liposomes derived from soya bean phospholipids in cosmetics.

Recently, water sensitive 20 to 30 micron -size microspheres of polymer structure have been developed for the delivery of fragrances, botanicals, and vitamins from anhydrous formulations, such as lipsticks, deodorants, antiperspirants and body sprays.

Liposomes are also used in anti-aging creams, treatment of hair loss. Liposome technology offers great opportunities for several new cosmetic products and that cosmetic developer would now have to deal very interestingly with raw material selection, characterization.

Marinosomes as Nutraceuticals:

Marine phospholipids can become an important marine ingredient in functional foods and have a high potential for use in products for human consumption, for use as delivery systems for drugs and in pharmacology [8].

Effective and low-cost anticancer combination drugs were developed from curcumin with other naturally occurring nutraceuticals (Choudhury et al., 2013). To benefit wholly from the effects of both $n-3 m$ 7PUFAs and curcumin, together nanomedicine with drug combination strategy will be used to propose a novel liposomal formulation of curcumin-loaded PUFAs liposomes.[11]

\section{CONCLUSION:}

Marinosomes are liposomes based on a natural marine lipid extract containing high ratio polyunsaturated fatty acids and can increase stability and increases circulation lifetime [t1/2] of drug which tends to deposit in the tissues. These vesicular systems containing marine lipids are more advantageous than liposomes for their stability and bioavailability.

\section{REFERENCES:}

1) Soumya saroj, doneyalex baby, s abitham. current trends in lipid-based delivery system and its applications in drug delivery 2012; 5:4-9.

2) International Scholarly Research Network ISRN Pharmaceutics Volume 2012, Article ID 474830, 14 pages doi:10.5402/2012/474830). 
3) Nageenarora, shilpiagawal, R.S. R murthy. latest technology advances in cosmeceuticals 2012;4(3):168-182.

4) F. Nactal,m.cansella, J.P.gouygou b, C gerbeaudc, P.meleard. Physical and chemical stability of marine lipid based liposomes under acidic condations .cooloids and B:biointerfaces .2001;20:257-266

5) F. nacckaa, m. canseiia, philippemeleard and Nicole combec, alstabnutrition et al. incorporation $\alpha$ tocopherol in marine lipid-based liposomes: in vitro and in vivo studies. lipids .2001;36:1313-1320

6) F.nacka, m. cansell, and Bernard entessangels . L8566 in lipids. 2001; 36:35-42

7) N.moussaoui, m.casell, A.denizot marinosomes, marine lipid -based liposomes: physical characterization and potential application in cosmetics international journal of pharmaceutics .2002:242:361-365.

8) Mozuratyer, rustard t, store. pro-oxidative activity of cod phosphor lipids in liposomes. European journal of lipid science techonology .2006;108:218-226

9) Maudscansella, n. moussaoui b, m.maneinib . prostaglandin $E_{2}$ AND INTERLUKIN -8 PRODICTION IN HUMAN EPI derimal keratinocytes exposed to marine lipid-based liposomes. international journal of pharmaceutics 2007; 342:277-280.
10) Joana Marcelina jos'eL.E.F limaasalettareisa , Carla matosb ,assessing the effects of surfactants on physical properties of liposomes membranes chemistry and physics of lipids. 2007; 146:94-103

11) Shimmaaibrhima, talsuakitagamia, toshihirokishib ,tetsuyaozekia. Curcumin marinosomes as promising nano drug delivary system for lung cancer.

12) Sawrniatasaraf , shailendarsaraf marinosomesapotential vesicular systems for drug delivary. journal of ravishanker university. 2010; 8;19.

13) Emilie gennia, gaetanewielgoszcollina, jean michelnjinakouea, e. velosatsya, jeanmichelkornpobsta, jenan-paul et. Al new trends in phosolipids class composition of marine sponges. comparative biochemistry and physiology part $b$ 2008: 150; 427-431.

14) United states patent beaudonin et al us 0006800299B1 patent ni; US6,800,299 2004; B1(5)

15) activities lenaburri, nilshoemls bastanobanni 2 and kjetilbergel marine omega -3 phospholipids: metabolism and biomolecular science $2012 ; 13$ : 15401-15419.

16) Jackie peters liposomal lypospheric health tips, nutritional supplements -2016 . 